\title{
Founders Group, International Association of Allergists
}

Abramson, Harold A., M. D. Alexander, Franz, M. D.

Bachman, Alois Eugenio, M. D. Bacigaluppi, Emilio Eduardo, M. D Bentolila, Leon, M. D. . Berggren, Bengt Georg, M. D. Bernton, Harry S., M. D. Black, William Byron, M. D.

(Deceased) Bohner, Caryle Bernard, M. D. Bordet, Jules, M. D. (Honorary) Bordet, Paul, Prof.

Dr. . Bozzola, Jose A., M. D. . Button, Cedric John Charles Bronfenbrenner, Jacques J., Ph. D.,

Dr. P. H. . . Brown, Ethan Allan, M. D. . Bucher, Karl, Prof. Dr.

Cadrecha, Alvarez, Jose, M. D. Castilla, Caupolican, M. D. . Clarke, T. Wood, M. D. . Coca, Arthur Fernandez, M. D., Crandall, Frank G., Jr., M. D.

Dahlberg, Gunnar, Prof. Dr. Davis, Ethel M., M. D. . Davison, Hal M., M. D. Dees, Susan

Coons, M. D. . Diaz Carrasco, Eduardo, M. D. Dias Da Costa, Paulo, M. D. Dohlman, Gösta, Prof. Dr. . Duchaine, Jacques Paul, M. D. Dumm, Jose Federico, M. D. Dutton, Loraine Orr, M. D.

Epstein, Stephan, M. D. Estrada de la Riva, Gonzalo, M

New York, N. Y. (U.S.A.) Chicago, Illinois (U.S.A.)

Buenos Aires, Argentina Buenos Aires, Argentina Rosario, Argentina Landskrona, Sweden

Washington D. C. (U. S. A.) Kansas City, Missouri (U. S. A.) Indianapolis. Indiana (U.S.A.)

Bruxelles, Belgium Bruxelles, Belgium Buenos Aires, Argentina London, England

St. Louis, Missouri (U. S. A.) Boston, Massachusetts (U. S. A.) Basle, Switzerland

Habana, Cuba Buenos Aires, Argentina Utica, New York (U.S.A.) (Honorary) Oradell, New Jersey (U.S.A.)

Los Angeles, California (U. S. A.)

Uppsala, Sweden

Chicago, Illinois (U. S. A.)

Atlanta, Georgia (U. S. A.)

Durham, No. Carolina (U. S. A.)

Santiago, Chile

Rio de Janeiro, Brazil

Lund, Sweden

Bruxelles, Belgium

La Plata, Argentina

El Paso, Texas (U.S.A.)

Marshfield, Wisconsin (U. S. A.) D. . Habana, Cuba

Farrerons-Có, Francisco J., M. D Faure, Ernesto, M. D. . Flores y de la Torre, Alberto, M. D. Forman, Jonathan, M. D. . Formijne, P., Prof. Dr. . Frankland, Alfred William, M. D. French, Sanford Williams, M. D. Frey, Walter, Prof. Dr. . Frouchtman, Raimundo, M. D. Frugoni, Cesare, M. D. .

Galvagno, Vicente J., M. D. Garat, Benigno Romulo, M. D. Gelfand, H. Harold, M. D. . Barcelona, Spain 
Cordoba, Argentina

Lima, Peru

Worthington, Ohio (U. S. A.)

Amsterdam, Holland

London, England

San Antonio, Texas (U. S. A.)

Berne, Switzerland

Barcelona, Spain

Rome, Italy

Buenos Aires, Argentina Buenos Aires, Argentina New York, New York (U. S. A.)

Founders Group

393

D.

Gigon, Alfred, Prof. Dr. Golz, Harold H., M. D. . Gottlieb, Philip, M. D. . Graham, William

Randolph, M Greco, Jose Bartholomew., M. D Groer, F., Prof. Dr. Grumbach, Arthur S., Prof.

Dr Gutmann, M. J., M. D. .

Hajos, K., Prof. Dr. . Hajos, Mary-Katharine, M. D. Halpern, Bernard N., Prof. Dr. Halpin, Lawrence James, M. D. Hanhart, Ernst, Prof. Dr. . Hansel, French K., M. D. . Hansen-Pruss, Oscar C, M. D. Harley, David, M. D. . Haxthausen, Holger, Prof. Dr. Hayden, Helen C, M. D. Henry, L. Dell, M. D. . Hooker, Sanford B., M. D. , Hughes, Robert F., M. D. . Hunter, R. B., M. D. .

Jadassohn, Werner, Prof. Dr. Jimenez Diaz, Carlos, Dr. .

Rabat, Elvin A., B. S., A. M., Ph. D

Kahn, I. S., M. D

Kallós, Paul, M. D

Kåmmerer, Hugo, Prof. Dr. Kaplan, Morris Aaron, M. D. . Knight, Granville F., M. D. . Koch, Carl Hjalmar Leonard, M. D Kohn, Cecil Mayor, M. D. . Kuhn, Hugh 1,M.D..

Leney, Fannie Lou, M. D. .

Levin, Samuel J

Lichtenstein, Adolf, Prof. Dr. (Deceased)

Löffler, Charles William, Prof. Dr.

Löfström, Gunnar, Prof.

Lutz, W., Prof. Dr

Macaulay, Douglas Blair, M. D. . Maclnnis, Katharine Baylis, M. D. Salazar-Malien, Mario, Dr. . Marrack, J. R., Prof. . Marsiaj, Nino, M. D. . . Moore, Merle W'., M. D. Meier, Rolf, Prof. Dr. . Ruiz, Moreno, Guido, M. D.

Olislagers, Hendricus Cornells, M. D.

Oliveira Lima, Antonio, M. D. .

Ordman, David, Dr

Basle, Switzerland Dhahran, Saudi Arabia Philadelphia, Penna. (U.S.A.) Richmond, Virginia (U. S. A.) Belo Horizonte, Minas, Brazil Krakow, Poland Zurich, Switzerland Jerusalem, State of Israel

Budapest, Hungary

Budapest, Hungary 
Paris, France

Cedar Rapids, Iowa (U. S. A.)

Zurich, Switzerland

St. Louis, Missouri (U. S. A.)

Durham, North Carolina (U. S. A.)

London, England

Copenhagen, Denmark

Chicago, Illinois (U. S. A.)

Ann Arbor, Michigan (U. S. A.)

Boston, Massachusetts (U. S. A.)

Hamilton, Ontario, Canada

Edinburgh, Scotland

Geneve, Switzerland Madrid, Spain

New York, New York (U. S. A.)

San Antonio, Texas (U. S. A.)

Helsingborg, Sweden

Miinchen, Germany

Chicago, Illinois (U. S. A.)

Santa Barbara, California (U. S. A.)

Lund, Sweden

Kansas City, Missouri (U. S. A.)

Hammond, Indiana (U. S. A.)

Oklahoma City, Oklahoma (U. S. A.; Detroit, Michigan (U. S. A.) Stockholm, Sweden Zurich, Switzerland Uppsala, Sweden Basle, Switzerland

Liverpool, England

Columbia, So. Carolina (U. S. A.)

Mexico

London, England

Brazil, South America

Portland, Oregon (U. S. A.)

Basle, Switzerland

Buenos Aires, Argentina

Amsterdam-Zuid, Netherlands Rio de Janeiro, Brazil Johannesburg, So. Africa

394

Founders Group

Peck, Samuel M., M. D. Peshkin, M. Murray, M.D. . Pipes, David McKowen, M. D. Prakken, ].

R., Prof. Dr. .

Quintero Fossas, Jose M., M. D

Rajka, Odon, Prof. Dr. . Ratner, Bret, M. D. Recarte, Pablo, Dr. Rich, Arnold Rice, M. D. .

Riley, Bernard Moreton, M. D. Rimington, C, Prof. Rockwell, George E., M. D. Roessle, Robert, Prof. Dr. . Rogers, Harry Lincoln, M. D. Rose, Bram, M. D. . Rosenow, Edward Carl, M. D.

Rowe, Albert Holmes, M. D. Rudolph, Jack A., M. D. Runnström, John, Ph. D., M. D

Prof.

D. 
Saenz, Abelardo, Prof. Dr. . Sanchez Medina, M., M. D. Sanders, Sam H., M. D. Sangiorgi, Piero, Prof. Dr. . Schick, Beta, M. D. Schiller, Irving William, M. D. Schonwald, Phillipp, M. D. (Deceased) Schwartz, Louis, M. D. . Sclafer, Jacques, M. D. . Service, William Corr, M. D. Shahon, Henry I., M. D. Shambaugh, George E., Jr., M. Solari, Miguel Agustín, M. D.

Southwell, Neville, M. D. . Spiegel, B, Edgar, M. D. Stoll, Arthur, Prof. Dr. .

Thomas, John Warrick, M. D. Tiselius, Arne Wilhelm Kaurin,

Prof. (Honorary) Tzanck, Arnault, M. D.

Unger, Leon, M. D.

Van Dishoeck, H.A.E., M. D. Vicencio, A. B., M. D. . Von Albertini, Ambrosius, Prof. Dr. Von Bergmann, Gustav, Prof. Dr.

Waldbott, George L., M. D Walker, Vera B., Dr. . Williams, D. A., M.D. . Withers, Orval R., M. D. Wittich, Fred W., M. D. Wolfer, Rudolph, Dr.

New York, New York (U. S. A.) New York, New York (U. S. A.) Asheville, No. Carolina (U. S. A.) Amsterdam, Holland

Habana, Cuba

Budapest, Hungary New York, New York (U. S. A.) Montevideo, Uruguay Baltimore, Maryland (U. S. A.) Sydney, New So. Wales, Australia London, England Milford, Ohio (U.S.A.) Berlin, Germany Philadelphia, Penna. (U.S.A.) Quebec, Canada Cincinnati, Ohio (U. S. A.) Oakland, California (U. S. A.) Miami Beach, Florida (U. S. A.) Stockholm, Sweden

Paris, France

Bogota, Colombia

Memphis, Tennessee (U.S.A.)

Milan, Italy

New York, New York (U. S. A.)

Boston, Massachusetts (U. S. A.)

Seattle, Washington (U. S. A.)

Washington, D. C. (U. S. A.)

Paris, France

Colorado Springs, Colorado (U.S.A.

Boston, Massachusetts (U. S. A.)

Chicago, Illinois (U.S.A.)

Buenos Aires, Argentina

London, England

New York, New York (U. S. A.)

Basle, Switzerland

Richmond, Virginia (U. S. A.)

Uppsala, Sweden Paris, France

Chicago, Illinois (U. S. A.)

Leiden, Holland Quezon City, Philippines Zurich, Switzerland München, Germany

Detroit, Michigan (U. S. A.)

Oxford, England

Cardiff, South Wales

Kansas City, Missouri (U. S. A.)

Minneapolis, Minnesota (U. S. A.)

Zurich, Switzerland 